\title{
Pericarditis and Necrotising Fascitis: Unusual Complications Following a Laparoscopic Appendectomy: A Case Report \\ Sumairanawaz Syed*
}

General Surgery Bedford hospital, South Wing, UK

\begin{abstract}
Appendicectomy is one of the most common surgical procedures performed. Published literature shows that laparoscopic appendicectomy is a safe procedure. A 35 year old female came to the hospital complaining of abdominal pain for the past few hours. History and examination revealed a classical case of acute appendicitis. The patient underwent laparoscopic appendicectomy the same night. Immediate post operative period was uneventful and she was discharged. But one day after her discharge she returned with vomiting, abdominal pain, swelling and tightness in the abdominal wall. Investigations revealed necrotising fasciitis and pericarditis. This case report shows that Laparoscopic appendicectomy can lead to unusual complications such as necrotising fascitis and pericarditis which cause significant postoperative morbidity. Therefore it can be concluded that it would be useful to use prophylactic antibiotics routinely preoperatively in cases of seemingly uncomplicated appendicitis to prevent infections like necrotising fascitis. It would also be beneficial to do preoperative ECG in all patients of suspected appendicitis so as to get a baseline ECG in persons with no known comorbidities.
\end{abstract}

\section{Introduction}

Appendicitis has always been amongst the most commonly encountered surgical emergencies. Therefore its treatment appendicectomy is one of the most common surgical procedures performed. Published literature shows that laparoscopic appendicectomy is a safe procedure [1]. With recent advances in surgery laparoscopic approach has become more common as compared to open appendicectomy. Open appendectomy had been associated with complications like: bleeding, wound infections, intra abdominal abscesses, visceral injuries leading to faecal fistula, intestinal obstruction, tubal infertility, right inguinal hernia, deep vein thrombosis \& clots to lung and other anaesthetic complications. These complications are much rarer with laparoscopic surgeries but laparascopic surgery has also been associated with some complications [2]. They include subtotal/incomplete appendicectomy, missed diagnosis, removal of a normal appendix, visceral injury-such as the small intestine, ureter or bladder leak at that edge of the colon where the appendix gets removed, haemmorrhage, infection of abdomen wound or blood, blood clot to the lungs and other anaeesthetic complications.

Some complications have been particularly associated with laparoscopic approach due to the instrumentation used and pneumoperitoneum created during laparoscopy. As a surgeon traverses the abdomen wall for laparoscopic access there is increased risk of skin infection, subcutaneous emphysema, haemorrhage, gas embolism and port site hernias [3].

The complications seen in this case are very rare complications of laparoscopic appendicectomy but their occurrence shows that laparoscopic appendicectomy can also be associated with dangerous complications that should be prevented by preoperative preventive measures if possible.

\section{Case Presentation}

A 35 year old lady previously well with no known comorbidities, presented with a one day history of sudden onset abdominal pain that had radiated from upper abdomen to right iliac fossa. It was sharp and continuous pain that had no aggravating and relieving factors. Pain was associated with an episode of vomiting. There was no fever, no loose stools/constipation and no urinary symptoms. On examination the patients abdomen was soft, non-distended with tenderness in the right iliac fossa, rebound tenderness was also present, rovsings sign positive and there was some guarding also. The diagnosis of acute appendicitis was made clinically.

Investigations showed a slightly raised white cell count $\left[12.7 \times 10^{\wedge} 9 / 1\right.$ with high neutrophil count $10.6 \times 10^{\wedge} 9 / \mathrm{L}$. The $\mathrm{C}$ reactive protein was 37 . Urine showed flecks of blood and pus and leukocytes ${ }^{+}$. The intravenous fluids were started and the patient was kept nil by mouth till laparoscopic appendicectomy was done. The technique used was open technique. 3 ports were used-umblical, left lower quadrant (left to the usual supra pubic) and right upper quadrant. No port site gas leak was noted during surgery and there was no excessive bleeding, trocar sizes and port site incisions matched appropriately.

The appendix had not perforated although it looked gangrenous at the time of surgery. The standard appendicectomy technique was used, the appendix base was cauterised and the appendix removed from umblical port. There was no peritoneal contamination noted. Post operatively no immediate complications occurred and the patient seemed recovering normally and therefore discharged. However just one day later she returned to the hospital with severe abdominal pain that she described as intermiittent to begin with, continuous later involving the left lower quadrant of the abdomen. On examination the abdomen was not distended. It was soft but tender along the port sites as expected but one of the port sites (left paraumblical) was reddish and seemed inflamed. She seemed generally unwell and was admitted and the initial query at the time of admission was for a pelvic abscess. She was kept nil by mouth, a naso gastric tube was put in. Intravenous fluid (normal saline $8 \mathrm{hr}$ ) was started. Intravenous antibiotics (augment $1.2 \mathrm{~g}$ $8 \mathrm{hr}$ and metronidazole $500 \mathrm{mg} 8 \mathrm{hr}$ ) were also started emperically. The

*Corresponding author: Sumairanawaz Syed, Clinical Fellow, General Surgery Bedford hospital, South Wing, UK, Tel: 07881275803; E-mail: sheebudeebu@hotmail.com

Received July 17, 2013; Accepted November 20, 2013; Published November 25,2013

Citation: Syed S (2013) Pericarditis and Necrotising Fascitis: Unusual Complications Following a Laparoscopic Appendectomy: A Case Report. Gen Med (Los Angel) 1: 124. doi: 10.4172/2327-5146.1000124

Copyright: (c) 2013 Syed S. This is an open-access article distributed under the terms of the Creative Commons Attribution License, which permits unrestricted use, distribution, and reproduction in any medium, provided the original author and source are credited. 
investigation reports showed a very high $\mathrm{C}$ reactive protein although the culture reports later showed that the pelvic fluid was not infected.

The X-Ray of the abdomen revealed dilated small bowel and her condition was thought to be due to post operative ileus. But during this admission she developed increased tightness in the skin of her lower left abdomen and oedematous swelling extending from her left loin to her left groin. The genitalia also swelled up. However no crepitus could be felt. The patient continued to have pain in abdomen \& she remained too ill to eat or drink anything by mouth (But she was mobile enough to go to the washroom and did not need to be catheterised). So a CT scan of abdomen was requested. The CT scan revealed small bowel obstruction up to terminal ileum and a collapsed colon. There was a small amount of ascitis and a very small amount of intraperitoneal gas. Within the left abdomen wall gas and fluid was found which was suspected to be phlegm caused by gas forming organism. Therefore the patient was monitored carefully for necrotising fascitis. The blood investigations were repeated. White cell count was 4 now against the initial value of 7.9. All other counts showed lower than the previous values. These changes were attributed to haemodilution. Therefore intravenous fluids were restricted. Same I/v antibiotics continued for a week. Analgesics included paracetemol $1 \mathrm{~g}$ i/v twice a day, tramadol orally three times a day, morphine as and when required basis, omeprazole was also given daily, cyclizine and metocloperamide were used to prevent vomiting, Low molecular weight Heparin was administered subcutaneously daily once. The patient opened her bowels 2 days later. Orals were started as tolerated, first fluids then light solids. By the end of the week she was passings tools in small amounts, could eat light diet only but did not have normal appetites still. The abdominal pain still persisted \& analgesia was needed to keep the patient comfortable all time. The tightness of skin started decreasing slowly after days of conservative management. In view of her continued abdomen pain an ECG was requested although she had no heart disease in the past and no history of any other medical illness. The medical registrar called onto review the ECG found it to be abnormal. Since the patient was not having any palpitations, no syncopal attacks, no chest pain, blood pressure $116 / 90$, pulse 86 , saturation of $94 \%$ on air and temperature 36.5 it was advised to repeat the ECG next day. Next day ECG was repeated and ST changes were found in leads V4-V6 (saddle shape) which the medical registrar said indicated previous pericarditis. An echo was booked to be done a few days later was the earliest available date. The patient remained on light diet and her abdomen was much softer but still tight in a left paraumblical region. Swelling subsided totally. But she suffered from bilious vomiting and was still passing stools in small amounts. A gastrograffin follow through was done and the report showed that the dye did not to reach the distal bowel but was present in the small bowel only and the patient also suffered from intermittent vomiting during this week although she passed wind but still passed stool in small amounts. These indicated the third complication i.e. intestinal obstruction/pseudo obstruction. Conservative management continued. A repeat AXR after 2 weeks was normal and patient requested discharge on request at the end of two weeks although she agreed to come back for an echo. She still needed analgesics four times a day for abdominal pain. Other Facts; Her weight was about $62 \mathrm{~kg}$ which was fine for her height (nearly 5,8 not obese at all). She was not on any regular medications, never had any medical problems and had no allergies. She was a non smoker and used to drink alcohol once in months at some functions not otherwise. She had travelled to Spain 1 week before the episode of appendicitis. She didn't take anything unusual there and was healthy during her stay. By occupation she was a police officer. Her grandfather was diabetic and had ischemic heart disease. Her parents did not have any significant medical history. This was the only time she had visited a hospital in her life.

\section{Discussion}

Post operative abdominal pain and vomiting can be due to Intestinal obstruction or ileus. The swelling and pain in abdomen wall can occur due to subcutaneous emphysema or necrotising fascitis. Subcutaneous emphysema is a known complication of laparoscopic surgery while necrotising fascitis has been a known complication of open appendicectomy. The complication of subcutaneous emphysema can occur in laparoscopy as insertion of surgical instruments can cause subcutaneous tissue layer injury. During peritoneal $\mathrm{CO}_{2}$ insufflation there can occur leakage of $\mathrm{CO}_{2}$ gas into these subcutaneous layers. This can extend as subcutaneous emphysema to parts of body beyond the surgical field which may lead to hypercarbia \& respiratory acidos is related to $\mathrm{CO}_{2}$ absorption. If not corrected, this in turn can cause hemodynamic changes, such as cardiac depression and arrhythmias [4]. However, the subcutaneous emphysema presents with a feeling of crepitus on palpation which was not there in this case. In this case the patient did not have respiratory acidosis and there was no crepitus. Necrotising fascitis on the other hand is a fast-spreading infection. It affects the fascia and spreads continuously if not controlled rapidly and causes secondary necrosis of the skin. It is usually associated with open surgery and gangrenous appendicitis. Very few cases have been previously described in association with laparoscopic appendicectomy so far and this case seemed to be one addition to this very rare complication of laparoscopic surgery as the patient did have tightness and swelling of abdominal wall and gases were seen in the abdominal wall in the CT images also although these resolved probably because of antibiotics [5]. But it can be seen from this case that laparoscopic surgery can cause morbidity due to complications such as intestinal obstruction, gas collection in abdominal muscles (necrotising fascitis) and a pericarditis!!!. Usually gas collection can occur following any laparoscopy but it usually resolves in a day or two. In this case it continued for weeks despite the patient being mobile and we continued antibiotics for 10 days. Also the initial redness of a port site and a gangrenous appendicitis favours the diagnosis of necrotising fascitis. The need for echo further complicated the scenario. It is not worthy to point out that pneumo pericardium is an extremely rare possible complication of laparoscopic surgery. The exact mechanism is not understood but embryologically the peritoneum communicates with the pericardium. May be the pneumoperitoneum created during laparoscopic access causes a pneumo pericardium by a transmission of high pressure that irritates the pericardium and causes pericarditis which resolves with time. In this case the ECG showed resolving pericarditis in a woman who had no previous heart problems and nothing else was there that could be considered as a cause of pericarditis. The patient went home on analgesics (4 times a day still needed) after a fortnight to come for echo later

\section{Conclusions}

The fact is that an echo can be normal in cases of active pericarditis also. So we concluded that ECG can be confirmatory evidence of a pericarditis that as occurred in this case as a complication of laparoscopic appendicectomy. A baseline ECG preoperatively would have added benefit but this was a $35 \mathrm{yr}$ old female, fit and healthy who never had any chest pain and there is no recommendation to do an ECG preoperatively in patients presenting as acute appendicitis at the age of 35. Being a female her pregnancy test was done which was negative but this case shows that it would have been beneficial to do a 
Citation: Syed S (2013) Pericarditis and Necrotising Fascitis: Unusual Complications Following a Laparoscopic Appendectomy: A Case Report. Gen Med (Los Angel) 1: 124. doi: 10.4172/2327-5146.1000124

baseline ECG which therefore should be done for all cases of suspected uncomplicated appendicitis also. It can also be concluded that it would be useful to use prophylactic antibiotics routinely preoperatively in cases of seemingly uncomplicated appendicitis to prevent infections like necrotising fascitis.

\section{References}

1. Dixon AR, Duff SE (2000) Laparoscopic appendicectomy;safe and useful for training. Ann R Coll Surg Engl 82: 388-391.
2. www.laparoscopyhospital.com/frequently_asked_question_abt_appendicitis.

3. Kavic MS, Kurt S (2006) Laparoscopic Appendectomy Prevention and management of laparoendoscopic Surgical Complications.

4. Yeoh CJ, Dawson C, Wiseman OJ (2006) Pneumopericardium: a rare complication of laparoscopic varicocoele ligation. Int Urol Nephrol 38: 671 . 672

5. Knos GB, Sung YF, Toledo A (1991) Pneumopericardium associated with laparoscopy. J Clin Anesth 3: 56-59. 